preprint

\title{
Study of the charge correlation function in one-dimensional Hubbard heterostructures
}

\author{
Y. Arredondo and H. Monien \\ Physikalisches Institut, Universität Bonn, Nussallee 12, 53115 Bonn, Germany
}

\begin{abstract}
We study inhomogeneous one-dimensional Hubbard systems using the density matrix renormalization group method. Different heterostructures are investigated whose configuration is modeled varying parameters like the on-site Coulomb potential and introducing local confining potentials. We investigate their Luttinger liquid properties through the parameter $K_{\rho}$, which characterizes the decay of the density-density correlation function at large distances. Our main goal is the investigation of possible realization of engineered materials and the ability to manipulate physical properties by choosing an appropriate spatial and/or chemical modulation.
\end{abstract}

\section{INTRODUCTION}

A key aspect of materials research is to find parameters to tune the physical characteristics of the system like conductivity and other desired properties. In the last decades there has been enormous progress in the generation of nanoscopic quasi-one-dimensional systems, e. g., carbon nanotubes [1, 2], semiconducting quantum wires [3, 4] and organic molecules [5]; as well as an intense study of their transport properties [6, 7, 8] such as superconductivity [9] and quantum Hall edge states [10, 11] . While the properties of homogeneous one dimensional systems (even with disorder) are relatively well understood, very little is known about the properties of strongly interacting inhomogeneous systems. Despite of the large effort in the study of heterostructures and quantum dots [12, 13, 14, 15, 16], there are still open questions which become relevant in modeling the transport through molecules, where the electrons interact strongly due to the reduced dimension. In addition, its chemistry induces potential barriers which alter the transport properties drastically. Technically it is very important to know how to control the transport and equilibrium properties. In this paper we present a detailed investigation of correlation effects in an inhomogeneous one-dimensional system including potential barriers.

The strong electron correlations, inherent to the lowdimensional structure, and the large quantum fluctuations induce new and interesting quantum phases. The relevant degrees of freedom are no longer the single particle electronic states but the collective spin and charge density waves. The low-energy electronic single particle excitations possesses vanishing spectral weight at the Fermi surface. The physics of such systems, in the homogeneous low-energy regime, is well described by the Tomonaga-Luttinger liquid (TLL) model [17, 18] introduced by Haldane [19]. Within this model, it is found that all correlation functions exhibit a power-law decay with the distance, which is specified only by the parameter $K_{\rho}$, known as the Tomonaga-Luttinger (TL) parameter.

For inhomogeneous structures the high-energy physics is determined by the underlying chemistry which, in the atomic scale, introduces Coulomb correlations and local potentials. On the other hand, at large length scales, the physics has to be described by the TLL model. In order to establish a connection between the low-energy TLL and the quasi-one-dimensional systems synthesized in the laboratory, we investigate the density-density correlation functions in the asymptotic region (i. e. for well separated positions $x$ and $x^{\prime}$ ). Position dependent on-site Coulomb interaction $U(x)$ and a local potential $V(x)$ are used to model the changes in the local chemistry of the heterostructures. This defines regions which, for slowly varying potentials, can be separately considered as homogeneous. We wish to study how the TL parameter changes close to the crossover regions. We expect to find a description of it in terms of $U(x)$ and the local density $n(x)$.

The paper is organized as follows: In Sec. III the composition of the investigated heterostructures is described and we plot our expectations in terms of the coupling parameters. In Sec. III we briefly recall the approximate results in the low-energy regime for correlation functions in the homogeneous case, and we describe the numerical procedure, the DMRG method, used to study the onedimensional heterostructures. The results are presented and discussed in Sec. IV] Finally we state our conclusions.

\section{HUBBARD HETEROSTRUCTURES}

The Hubbard heterostructures we investigate are chains with a length of $L$ sites and on-site Coulomb interaction $U$, which switches between two different values. In our case it can be visualized as a valley around the middle of the chain with sharp edges at the sites labeled $x_{L}$ and $x_{R}$. We will refer to this system as Heterostructure $I$. We expect that the slight discontinuity in the charge distribution, caused by this form of interaction, will not strongly affect the correlation between the adjacent regions and will make it possible to find a TLL behavior, even in the region after the change in the $U$ interaction. On a second heterostructure (called from here on Heterostructure $I I$ ), in addition to the Coulomb interaction described, two potential walls are introduced through the confining potential $V(\gg U)$. Because of the sharp discontinuity in the charge distribution, we do not expect to find a TLL extending beyond the point of the scattering 
potential, however it might still be possible to approximate the TLL in the different subchains, since in each of them we expect to find a homogeneous particle distribution. Fig. 1 shows the layout of the heterostructures.

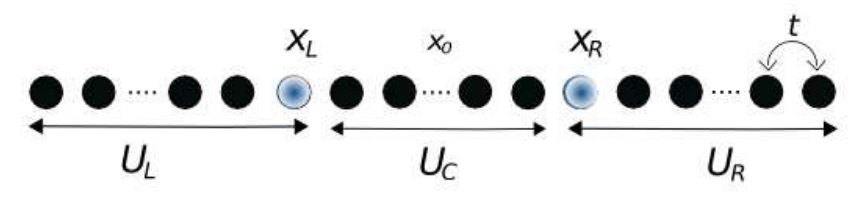

FIG. 1: General arrangement of a Hubbard heterostructure. The measurements for $\left\langle n(x) n\left(x_{0}\right)\right\rangle$ were carried out from the middle point $x_{0}=120$.

Our starting point is an inhomogeneous form of the Hubbard Hamiltonian:

$$
H=-t \sum_{i, \sigma} c_{i, \sigma}^{\dagger} c_{i+1, \sigma}+\sum_{i} U_{i} n_{i \uparrow} n_{i \downarrow}+\sum_{i, \sigma} V_{i} n_{i \sigma}
$$

where $c_{i, \sigma}^{\dagger}\left(c_{i, \sigma}\right)$ is the creation (annihilation) operator with spin $\sigma(=\uparrow, \downarrow)$ on the site $i$ and $n_{i \sigma}=c_{i, \sigma}^{\dagger} c_{i, \sigma}$ is the electron number operator. $t=1$ is the nearest neighbor hopping matrix, which we choose to set the energy scale. The Hamiltonian in Eq. (1) incorporates the different systems we want to study and will allow us to find out if such systems resemble a TLL and, in that case, also to determine the $K_{\rho}$ parameter from the density-density correlation function.

The sites $x_{L}$ and $x_{R}$ divide the whole system into three homogeneous subchains $U_{L}, U_{C}$ and $U_{R}$, raising two questions: first, how the charge correlation function behaves in the whole system and second, whether the known results for the homogeneous regime can be recovered within the subchains.

\section{APPROXIMATE DESCRIPTION OF ONE-DIMENSIONAL SYSTEMS IN THE LOW-LYING ENERGY SECTOR}

The low-lying energy, long-distance physics of onedimensional fermionic systems is described by bosonic collective excitations. This bosonization technique yields an exact solution for the TL model, whose complete description depends solely on the charge and spin velocities and the TL parameter $K_{\rho}$. In the next subsections we first briefly recall the known results [20, 21] for the density correlation function in the case of homogeneous systems and explain in detail the numerical method used to measure the correlation functions in the inhomogeneous systems.

\section{A. Homogeneous regime}

In a homogeneous TLL, $K_{\rho}$ determines the longdistance decay behavior of all the correlation functions. In the absence of external magnetic field or spin anisotropic interactions, the charge correlation function is given by

$$
\begin{array}{r}
\langle n(x) n(0)\rangle=\frac{K_{\rho}}{(\pi x)^{2}}+\frac{A_{1} \cos \left(2 k_{\mathrm{F}} x\right)}{x^{1+K_{\rho}}} \ln (\mathrm{x})^{-\frac{3}{2}} \\
+\frac{A_{2} \cos \left(4 k_{\mathrm{F}} x\right)}{x^{-4 K_{\rho}}}+\ldots
\end{array}
$$

Even though the constant coefficients $A_{1}, A_{2}$, and $B_{1}$ depend on the model, the algebraic decay is characterized only by $K_{\rho}$. Of special physical interest are the charge density waves with wave vectors $2 k_{\mathrm{F}}$ and $4 k_{\mathrm{F}}$. While the $2 k_{\mathrm{F}}$ mode dominates over the $4 k_{\mathrm{F}}$ for $K_{\rho} \geq \frac{1}{3}$, for sufficiently large values of the on-site Coulomb interaction $U$, the $4 k_{\mathrm{F}}$ charge mode dominates over the $2 k_{\mathrm{F}}$ mode.

As a test for our numerics, we considered the case of a homogeneous chain for which we confirmed the results obtained from the Bethe Ansatz [20, 22] for the correlation functions. In Fig. 2 we show our results for several values of $U$ obtained with a homogeneous chain of length $L=240$ sites. We will use this form of the density correlation function to analyze the low-energy behavior of the Hubbard heterostructures.

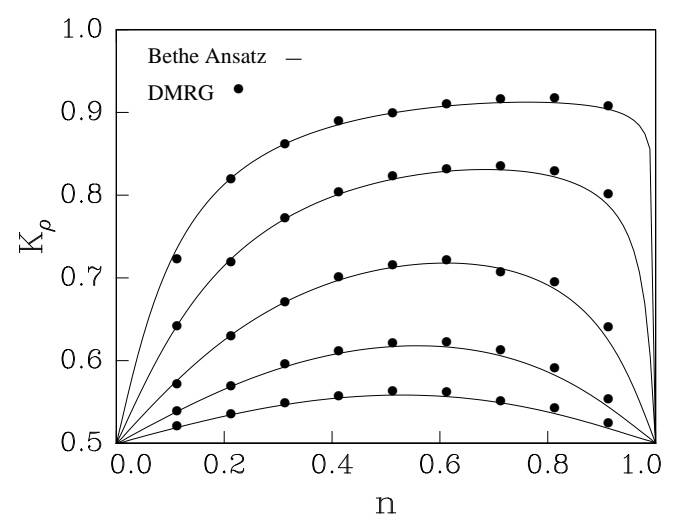

FIG. 2: Tomonaga-Luttinger parameter values for the Hubbard lattice compared to our numerical evaluation of $K_{\rho}$ (dots). $U=1.0,2.0,4.0,8.0,16.0$ from top to bottom.

\section{B. Inhomogeneous regime Numerical study}

The measurement of observables, which include ground state energies and correlation functions, is carried out using the density matrix renormalization group (DMRG) [23, 24, 25], a method whose roots go back to the numerical renormalization group formulated by Wilson [26]. The DMRG is an efficient numerical method developed 
to overcome the intrinsic difficulties of low-dimensional strongly interacting systems.

The DMRG provides two algorithms to handle an otherwise exponentially-increasing Hilbert space of a manybody system. Both implementations, finite-size and infinite-size DMRG base, as in Wilson's renormalization group, on a blocking treatment of a lattice system in realspace, whose basis of the corresponding Hilbert space is decimated under a certain criterion. In the renormalization group procedure, the decimation of the system's basis is done by selecting $m$ states with the lowest energy eigenvalues to obtain the ground state of a system. This proved to be a reliable method to solve systems, such as the Kondo problem, for which the coupling between successive sites decreases exponentially. Thus, it was plausible to ignore the connections between neighboring blocks, setting to 0 the wave function at the sites outside of the block of interest. This lead to inaccuracies when studying systems such as the Hubbard model, where there is no intrinsic separation of the energy scales. To solve this, White proposed other criteria to handle both the boundary conditions when adding a new site to the system as well as the selection of states to best represent it.

The DMRG method considers the system to be connected to a bath, which is a second block, forming in total a superblock. The interactions between the system and the bath set the boundary conditions at the edge sites of the system as if it would be part of a larger system. In this way, the procedure becomes more accurate as the system gets larger. The wave function in the superblock has the form $|\Psi\rangle=\sum_{i, j} \Psi_{i j}|i\rangle \otimes|j\rangle$, where $i$ are the states on the system and $j$ are those on the bath. From this, the reduced density matrix of the system is $\rho_{i i^{\prime}}=\sum_{j} \Psi_{i j} \Psi_{i^{\prime} j}$. The crucial point is that the density matrix contains all the information needed to calculate any property of the system and so, the state of the system can be optimally represented by keeping the $m$ most probable states given from the density matrix of the system.

We use the finite-size DMRG algorithm. This consist of the following steps: After growing our system up to a fixed size $L$, by means of the infinite-size DMRG, the basis of this final system is optimized to best represent the desired target state, like the ground state, by sweeping through the system repeatedly. A sweep over the system is an iterative process which starts with a small block on the right extreme of the chain. This is grown to in the left direction by adding a site to the right block and connecting it to a bath or environment on the left side. The environment information was collected from the infinitesize algorithm. The total size of the system is always kept constant. As soon as the decreasing size of the left block reaches a single site the procedure is stopped. We save the information of the right blocks and can use it now to start a similar procedure with a block on the left side of the chain being grown in the right direction. This procedure is repeated until convergence is reached.

With each step, the chain grows one site in the current direction, and the basis of the new system must be truncated to keep the Hilbert space manageable. All the necessary operators are transformed and stored every time this happens. With every step, the choice of states in the truncation of the basis becomes a better representation of the system. This leads to an optimal truncated basis for representing the target state on the finite system. After convergence was reached, we can proceed to measure other observables.

The numerical error caused by truncation of the original basis can be measured through the weight of the states that were discarded in a DMRG step. Our systems, with $L=240$ sites under open boundary conditions, were investigated keeping $m=256$ density-matrix states, rendering a maximum truncation error of approx. $10^{-6}$.

\section{Density-density correlation function}

An operator $A$, acting either on the left or on the right block, can be written in the basis of the specific block as $\langle\Psi|A| \Psi\rangle$. In the case of correlation functions like $\langle\Psi|A B| \Psi\rangle$, handling operators requires some extra attention. The operators $A$ and $B$ can operate either on equal or on different blocks. The last case may lead to errors in the calculation of the expectation value of the product $A B$, since each operator is separately written in its corresponding basis. The way to proceed is to build the exact operator $C=A B$, in a full basis from the beginning and transform it as is done for the rest of the operators.

We calculated the TL parameter $K_{\rho}$ by measuring the correlation function between the sites $x$ and $x_{0}$ : $C_{x}=\left\langle n(x) n\left(x_{0}\right)\right\rangle-\langle n(x)\rangle\left\langle n\left(x_{0}\right)\right\rangle$, where the static expectation values were subtracted. To reduce the effect of the local density oscillations, we take the average over pairs of correlation functions for neighboring sites calculating $C(r)=\left(C_{x}+C_{x+1}\right) / 2$, with $r=\left|x-x_{0}\right|$ and $x_{0}$ in the middle point of the chain. Due to the symmetry of the problem we can, in principle, choose either branch of the system to estimate $K_{\rho}$.

\section{RESULTS}

Using systems with open boundary conditions, finite size effects are induced. One example of these effects are the local density oscillations and the charge accumulation close to the edges of the system, shown in Fig. 3. The charge distribution is expected to be symmetric around the middle of the chain. We observe, however, that the symmetry is slightly perturbed, as seen in Fig. 3, at the positions where the Coulomb potential switches values. For our purposes, such small changes are negligible, specially after taking the average over pairs of correlation functions, as explained in Sec. III. It is still observed 
that the charge density remains fairly homogeneous in the valley of the Coulomb interaction.

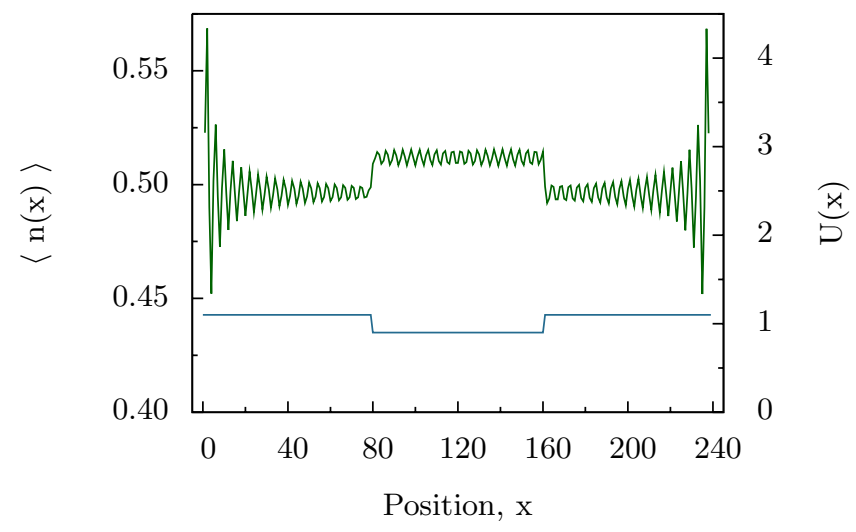

FIG. 3: Density profile $\langle n(x)\rangle$ for Heterostructure I, where the on-site Coulomb potential (bottom line on the graph with scale on the right) is $U_{L}=U_{R}=1.1$ and $U_{C}=0.9 . \quad V(x)=$ 0.0 for all sites. The band filling is $n=0.5$.

To estimate $K_{\rho}$, we fit the values of the numerical data to the leading term of Eq. (2), $K_{\rho} / \pi^{2} r^{2}$, leaving aside, at least for the moment, the logarithmic corrections. In figures 5 and 6 the density-density correlations are shown for both heterostructures and for band fillings of $n=$ 0.20 and $n=0.50$. We will refer to the region from the middle of the chain up to the boundary (at the site $x_{R}$ ) of the Coulomb valley as the region $R_{1}$, and from this point until the end of the chain as the region $R_{2}$. In the following we describe with detail the results for each heterostructure.

Density-density correlation functions for Heterostructure I. As a first structure we take a slightly inhomogeneous Hubbard lattice setting $U_{L}=U_{R}=1.1$ and $U_{C}=0.9$ with $V(x)=0.0$ for all sites. The valley in the on-site Coulomb repulsion has sharp edges at the sites $x_{L}$ and $x_{R}$, as shown in the Fig. 3. This however, and as seen from the full line in both Fig. 5] and in Fig. 6. does not alter significantly the continuous decay of the correlation function. For all band fillings, $0.10 \leq n \leq 1.0$, the power-law decay extends beyond the boundary point and is not completely constrained to any of the regions $R_{1}$ or $R_{2}$. In Fig. [7] the values for the TL parameter are shown as a function of the band filling. We observe that $K_{\rho}<1.0$, which indicates that spin or charge density waves are present. The $2 k_{\mathrm{F}}$ oscillations can be also observed in the graphs and a closer view is presented in Fig. 4. A fitting of the $2 k_{\mathrm{F}}$ oscillations succeeded over the whole system only for $n \leq 0.5$. In the case of $n>0.5$, the fitting of the data was only successful at large distances. This behavior is reflected on the values of $K_{\rho}$, as we observe in Fig. 7 the two different values sets for the density intervals already mentioned. With this we confirmed the power-law decay of the correlation functions as it was possible to determine $K_{\rho}$ also including the first logarithmic correction.

We compared the results with a similar configuration, this time with an interaction of the form $U(x)=\cos (\alpha x)$ with $\alpha$ a constant. The valley around the center of the system remains but the transition on the potential towards the ends is done in a smoother way. This variation of $U$ resulted in the same values for the correlation functions as already presented. showing that the sharp edges of the on-site potential did not influence strongly the Luttinger liquid behavior of the system.

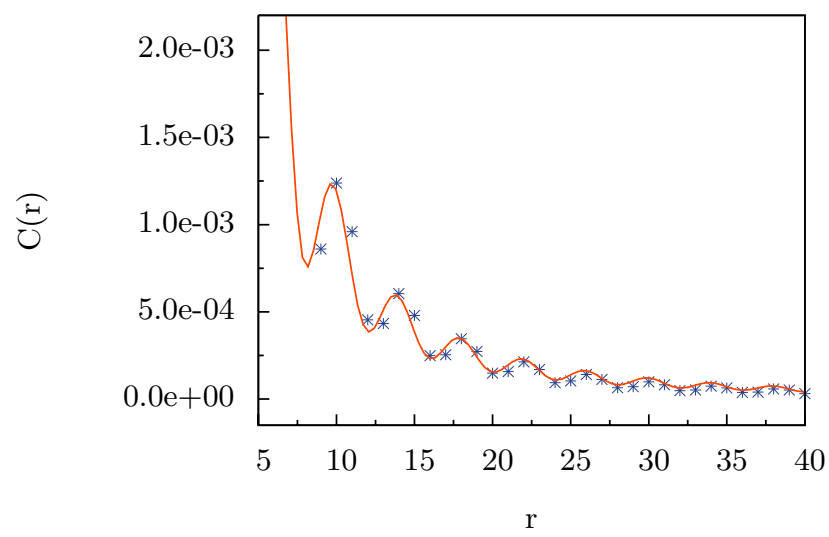

FIG. 4: Density-density correlation function for Heterostructure $I$ with $n=0.50$. The crosses show the numerical data and the solid line result after fitting including the term $A_{1} \cos \left(2 k_{\mathrm{F}} x\right) x^{-\left(1+K_{\rho}\right)} \ln (\mathrm{x})^{-\frac{3}{2}}$.

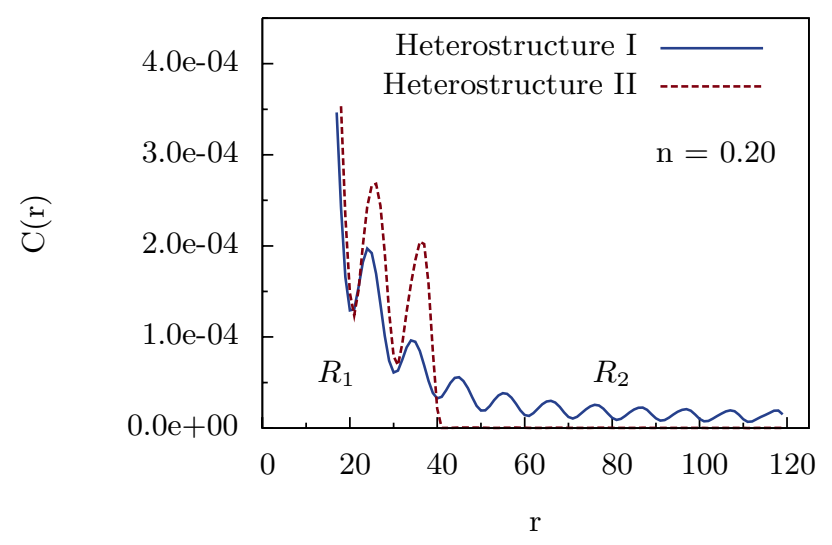

FIG. 5: Density-density correlation function for Heterostructures $I$ (continuous line) and II (broken line). In the first case we found $K_{\rho}=0.864$. In the second case, $K_{\rho}=1.093$ only within $R_{1}$, with $n=0.20$ in both cases.

Density-density correlation functions for Heterostructure II. In this case we keep the valley in the Coulomb interaction of the former case: $U_{L}=U_{R}=1.1$ 


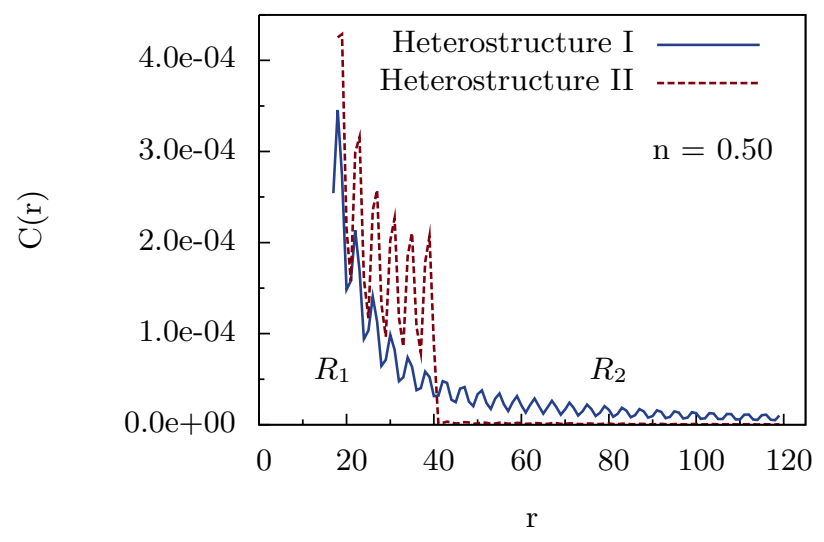

FIG. 6: Density-density correlation function for Heterostructures $I$ (continuous line) and $I I$ (broken line). In the first case we found $K_{\rho}=0.838$. In the second case $K_{\rho}=1.15$ only within $R_{1}$, with $n=0.50$ in both cases.

and $U_{C}=0.9$. Furthermore we simulate two potential walls by introducing the confining potential $V_{x_{L}}=$ $V_{x_{R}}=10.0$ and $V(x)=0.0$ for the rest of the sites. The results in the case of the Heterostructure II distinguished strongly from those previously described. The introduction of the confining potential $V\left(x_{L}\right)$ and $V\left(x_{R}\right)$ generated stronger changes from one region to the other, killing the oscillations beyond the $x_{R}$ point. In Fig. 5 and Fig. 6 the broken line shows how the decay of the correlation function is abruptly interrupted by the scattering potential, not having further space to fully establish the decay in the amplitude of the $2 k_{\mathrm{F}}$ oscillations. We found however, that even in the constrained region $R_{1}$ the correlation function obeys a power law decay with $K_{\rho}>1.0$, which is indicative of a Fermi liquid behavior.

\section{CONCLUSIONS}

In this paper we have investigated the behavior of density correlation functions in one-dimensional heterostructures. We described how junctions between different types of atoms influence the variation in space of the TL parameter. The heterostructures as defined can be seen as unions of subunits with different coupling constants in which the TLL for homogeneous systems is to be expected. However, our findings show that a slow variation of the on-site Coulomb potential, as in the first case, does not interrupt nor split the decay of the densitydensity correlation functions between the regions and the system as a whole behaves as a TLL. Similar systems were investigated [16] where the on-site Coulomb potential was turned on and off over the subchains. For such systems an effective exponent, $K_{\rho}^{*}=f\left(K_{1, \rho}, K_{2, \rho}\right)$, was calculated considering, for example, two subchains which were assumed as independent, homogeneous TLL's. Us-

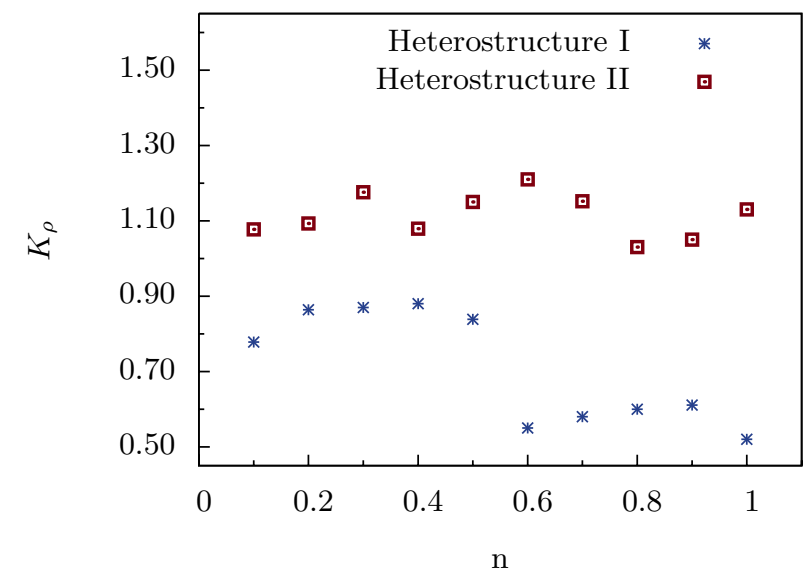

FIG. 7: TL parameter $K_{\rho}$ for both heterostructures as a function of the band filling. For Heterostructure $I I$, only within the region $R_{1}$. For Heterostructure $I K_{\rho}<1.0$ indicating a Luttinger liquid behavior. In the second heterostructure $K_{\rho}>1.0$, which is the benchmark of a Fermi liquid.

ing DMRG, their reported values could only be partially reproduced, namely for densities $n<0.6$.

We have also found a completely different behavior resulting from the introduction of a scattering potential $V$ at the junctions between the subunits, as done for the systems in the second case. Our findings in such case show that the TLL is not a universal feature for onedimensional systems. Concerning the dynamics in heterostructures, further work remains to be done. Transport properties at temperatures different from zero are a key in the construction of properly tunable electronic devices.

We wish to acknowledge useful discussions with A. Millis and thank C. Kollath for a helpful revision of the paper.
[1] S. Iijima and T. Ichihashi, Nature 363, 603 (1993).

[2] H. Ishii, H. Kataura, H. Shiozawa, H. Yoshioka, H. Otsubo, Y. Takayama, T. Miyahara, S. Suzuki, Y. Achiba, M. Nakatake, et al., Nature 426, 540 (2003).

[3] S. Tarucha, T. Honda, and T. Saku, Solid State Communications 94, 413 (1995).

[4] O. M. Auslaender, A. Yacoby, R. de Picciotto, K. W.
Baldwin, L. N. Pfeiffer, and K. W. West, Science 308, 88 (2005).

[5] K. Bechgaard, C. S. Jacobsen, K. Mortensen, H. J. Pedersen, and N. Throup, Solid State Communications 33, 1119 (1980).

[6] O. M. Auslaender, A. Yacoby, R. de Piccioto, K. W. Baldwin, L. N. Pfeiffer, and K. W. West, Phys. Rev. 
Lett. 84, 1764 (2000).

[7] M. Bockrath, D. H. Cobden, J. Liu, A. G. Rinzler, R. E. Smalley, L. Balents, and P. L. McEuen, Nature 397, 598 (1999).

[8] L. Degiorgi and D. Jerome, J. Phys. Soc. Jpn 75, 051004 (2006).

[9] D. Jerome, A. Mazaud, M. Ribault, and K. Bechgaard, J. Physique Lett. 41, 95 (1980).

[10] F. P. Milliken, C. P. Umbach, and R. A. Webb, Solid State Communications 97, 309 (1996).

[11] A. M. Chang, Rev. Mod. Phys. 75, 1449 (2003).

[12] K. Hallberg, A. A. Algia, A. P. Kampf, and B. Normand, Phys. Rev. Lett. 93, 067203 (2004).

[13] C. J. Gazza, M. E. Torio, and J. A. Riera, Phys. Rev. B 73, 193108 (2006).

[14] C. A. Büsser, A. Moreo, and E. Dagotto, Phys. Rev. B 70, 035402 (2004).

[15] S. Ejima, F. Gebhard, and S. Nishimoto, Phys. Rev. B 74, 245110 (2006).
[16] J. Silva-Valencia, E. Miranda, and R. R. dos Santos, Phys. Rev. B 65, 115115 (2002).

[17] J. M. Luttinger, Phys. Rev. 119, 1153 (1960).

[18] S. Tomonaga, Prog. Theor. Phys. 5, 544 (1950).

[19] F. D. M. Haldane, J. Phys. C: Solid State Physics 14, 2585 (1981).

[20] H. J. Schulz, Phys. Rev. Lett. 64, 2831 (1990).

[21] T. Giamarchi and H. J. Schulz, Phys. Rev. B 39, 4620 (1989).

[22] F. G. S. Ejima and S. Nishimoto, Europhys. Lett. 70, 492 (2005).

[23] S. R. White, Phys. Rev. Lett. 69, 2863 (1992).

[24] S. R. White, Phys. Rev. B 48, 10345 (1993).

[25] R. M. Noack and S. R. White, in Density Matrix Renormalization: A New Numerical Method in Physics (Springer, Lecture Notes in Physics, Vol. 528, Berlin, 1999).

[26] K. G. Wilson, Rev. Mod. Phys. 47, 773 (1975). 